\title{
Equivalent and Inequivalent Formulations of Classical Mechanics*
}

\author{
Thomas William Barrett
}

\begin{abstract}
In this paper, I examine whether or not the Hamiltonian and Lagrangian formulations of classical mechanics are equivalent theories. I do so by applying a standard for equivalence that was recently introduced into philosophy of science by Halvorson $(2012,2016)$ and Weatherall (2016a). This case study yields three general philosophical payoffs. The first is a specific plea for clarity in our discussions of equivalence, while the second and third concern how we should interpret what our physical theories say about the world.
\end{abstract}

\section{Introduction}

There is sometimes a sense in which two physical theories are equivalent. A particularly famous case of this arose in the early years of quantum mechanics. In brief, this story goes as follows:

There were once two formulations of quantum mechanics. Heisenberg had his theory of matrix mechanics, and Schrödinger had his theory of wave mechanics. It seemed that there was a substantive choice to be made between these two approaches. They used different and seemingly incompatible mathematical apparatus, and therefore appeared to be distinct theories. Both matrix mechanics and wave mechanics accounted equally well for the empirical data, however, so choosing between them proved particularly difficult, and the debate quickly became acrimonious. Schrödinger (1926) was "discouraged" and "repelled" by Heisenberg's theory and argued that it "lacked visualizability." Heisenberg found Schrödinger's theory "repulsive" and claimed that "what Schrödinger writes about visualizability [...] is crap" (Heisenberg, 1979).

*Thanks to Neil Dewar, Ben Feintzeig, Jim Weatherall, JB Manchak, Noel Swanson, Alex Meehan, and Jeff Barrett for helpful comments and discussion. This material is based upon work supported by the National Science Foundation under Grant No. DGE 1148900. 
The dispute between Heisenberg and Schrödinger continued until von Neumann (1932) showed that there is a sense in which matrix mechanics and wave mechanics are actually equivalent theories. ${ }^{1}$ We realized that there was no substantive choice to be made between these two formulations of quantum mechanics after all. They were the same theory, just presented in two different guises.

Matrix mechanics and wave mechanics are not the only theories that are thought to bear this kind of relationship to one another. Indeed, in this paper, I will consider a pair of theories that has recently received attention from philosophers of physics: Hamiltonian mechanics and Lagrangian mechanics. ${ }^{2}$ These two theories are geometric formulations of classical mechanics. In the Hamiltonian formulation, the state of a system is specified by its position and momentum, while in the Lagrangian formulation, the state of a system is specified by its position and velocity. The Hamiltonian formulation uses the mathematical apparatus of cotangent bundles and Hamiltonians, while the Lagrangian formulation uses the mathematical apparatus of tangent bundles and Lagrangians. But these are normally taken to be nominal differences between the two theories. They may be formulated in different languages, but the standard view among physicists and mathematicians is that Hamiltonian mechanics and Lagrangian mechanics are nonetheless - like matrix mechanics and wave mechanics equivalent theories.

Unfortunately, the standard view about Hamiltonian and Lagrangian mechanics is rarely made precise. Textbooks on classical mechanics often assert that the two formulations are equivalent, but they generally do so without saying what it might mean for two theories to be equivalent. ${ }^{3}$ North (2009) and Curiel (2014) have therefore taken the opportunity to dissent from the standard view. They argue that it is mistaken, and that Hamiltonian and Lagrangian mechanics are actually inequivalent theories.

There has been a flurry of recent work - much of which was directly inspired by North and Curiel's discussions of classical mechanics - developing tools for the purpose of answering questions of equivalence between theories. ${ }^{4}$ My aim in this paper is to apply these tools to the case of Hamiltonian and Lagrangian mechanics. We therefore begin by reviewing the recent work on equivalence. We discuss a number of different standards of equivalence that have been proposed, and in doing so, clarify what it might mean for two theories to be equivalent. We then turn to the case of Hamiltonian and Lagrangian mechanics and apply a particularly promising standard of equivalence, called categorical equivalence, which was recently introduced into philosophy of science by Halvorson (2012, 2016) and Weatherall (2016a). I show that categorical equivalence allows one

\footnotetext{
${ }^{1}$ See Casado (2008) and the references therein for further details.

${ }^{2}$ See North (2009), Halvorson (2011), Swanson and Halvorson (2012), Curiel (2014), Barrett (2015), and Teh and Tsementzis (2017).

${ }^{3}$ For example, see the discussion in Abraham and Marsden $(1978, \S 3.6)$.

${ }^{4}$ In addition to the papers already cited, see Andréka et al. (2005), Barrett and Halvorson (2016a,b, 2017a,b), Coffey (2014), Halvorson (2013), Glymour (2013), Hudetz (2015, 2016), Knox (2011, 2014), Rosenstock et al. (2015), Rosenstock and Weatherall (2016), Tsementzis (2015), Van Fraassen (2014), and Weatherall (2017). See also the classic work by Glymour (1971, 1977, 1980), Quine (1975), and Sklar (1982).
} 
to capture the standard view that Hamiltonian and Lagrangian mechanics are equivalent. But depending on precisely how one understands the two theories, categorical equivalence also leaves room for one to follow North and Curiel and consider Hamiltonian and Lagrangian mechanics inequivalent.

This case study of equivalence in classical mechanics yields three general philosophical payoffs. First, we will see that questions of equivalence are particularly sensitive both to the standard for equivalence that one employs, and to the precise way in which one understands the theories in question. Whether one considers Hamiltonian and Lagrangian mechanics to be equivalent depends crucially on what one means by "equivalent," "Hamiltonian mechanics," and "Lagrangian mechanics." This first payoff is primarily a plea for clarity in our discussions of equivalence, but the second and third payoffs have more philosophical bite. They concern the question of how we should interpret - or unravel the content of - our physical theories. I argue that this question is closely related to questions of equivalence like the one under consideration in this paper. The tools we use to answer questions of equivalence can also be used when we interpret our physical theories. The second payoff suggests that these tools help us to rule out certain unsatisfactory methods of interpreting our physical theories, while the third payoff suggests that they also point us to better methods. The moral of these two payoffs is that we should use cases of equivalent theories as a guide for how best to interpret our physical theories.

\section{When are two theories equivalent?}

Before turning our attention to the specific case of Hamiltonian and Lagrangian mechanics, we need to ask a more general question: Under what conditions should we consider two theories equivalent?

One way that philosophers of science have approached this question is by proposing different formal standards of equivalence. One such criterion is employed by North (2009) in her discussion of classical mechanics. She argues that Hamiltonian and Lagrangian mechanics are inequivalent because models of the two theories are not isomorphic; there are "differences in structure" between them (North, 2009, p. 72). ${ }^{5}$ North's treatment of classical mechanics points to the following general standard of equivalence between theories.

Criterion. Theories $T_{1}$ and $T_{2}$ are equivalent according to the model isomorphism criterion if for every model of $T_{1}$ there is an isomorphic model of $T_{2}$, and vice versa.

North implicitly relies on something like the model isomorphism criterion when she infers the inequivalence of Hamiltonian and Lagrangian mechanics from the differences in structure between models of the two theories. She is not

\footnotetext{
${ }^{5}$ More precisely, North argues that models of Hamiltonian mechanics have "less structure" than models of Lagrangian mechanics, which implies that they are not isomorphic. See North $(2009, \S 3)$ for a detailed expression of her view, and Halvorson (2011), Swanson and Halvorson (2012), Curiel (2014), and Barrett (2015) for responses.
} 
the only proponent of this standard. ${ }^{6}$ For example, it has recently been argued by Halvorson (2012) that adherents to the popular "semantic view of theories" are forced to endorse this standard of equivalence. ${ }^{7}$ And regardless of one's views on the debate between the semantic and syntactic views of theories, the basic intuition behind the model isomorphism criterion is certainly tempting. If there is a sense in which the mathematical structure of a theory's models directly mirrors the structure of the world, then theories whose models are isomorphic must "say the same thing" about the world. One might say, for example, that they "ascribe the same structure" to the world. Conversely, theories whose models are not isomorphic must "say different things" about the world.

Despite this appealing intuition, the model isomorphism criterion suffers from a serious shortcoming: It judges too many theories to be inequivalent. Indeed, it seems to make the wrong verdict when applied to the classic example of equivalent theories. Since a matrix algebra is not isomorphic to a space of wavefunctions, the model isomorphism criterion judges Heisenberg's matrix mechanics and Schrödinger's wave mechanics to be inequivalent theories. Although this is certainly an unsettling verdict, I take the following example to be even more decisive. $^{8}$

Example 1. It is well known that there are different ways to formulate general relativity. For example, it can be formulated on a smooth manifold with metric of signature $(1,3)$, and it can be formulated on a smooth manifold with metric of signature $(3,1)$. One can picture the models of these two theories as follows:

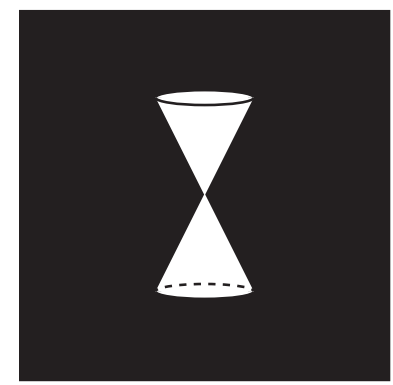

(1-3) general relativity

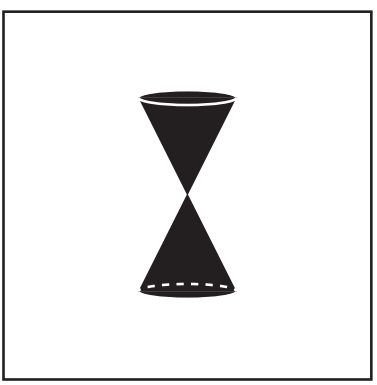

(3-1) general relativity

There is a strong sense in which these two formulations of general relativity are

\footnotetext{
${ }^{6}$ Curiel argues that Hamiltonian and Lagrangian mechanics are inequvialent because "the underlying geometrical structures one uses to formulate each theory are not isomorphic" (Curiel, 2014, p. 1). This might sound like an endorsement of the model isomorphism criterion, but he later makes clear that he does not have this standard in mind. Curiel's conclusion is instead rooted in the idea that these geometric structures have different dynamical and physical significance for the two theories. See (Curiel, 2014, p.297-9) and the discussion surrounding our Proposition 3 in what follows.

${ }^{7}$ For additional discussion see Halvorson (2013), Glymour (2013), and Van Fraassen (2014).

${ }^{8}$ This example appears in Barrett (2015) to illustrate a slightly different point. Additional examples of undesirable verdicts made by the model isomorphism criterion are offered by Halvorson (2012).
} 
equivalent. Indeed, they only differ with respect to a choice of sign convention (i.e. a choice of whether to "color" the insides of the lightcones white or black). ${ }^{9}$

These two formulations of general relativity are inequivalent according to the model isomorphism criterion. Manifolds with metric can only be isomorphic if their metrics have the same signature. Since models of these two formulations of general relativity employ metrics of different signatures, they are not isomorphic, and the two theories are therefore inequivalent according to the model isomorphism criterion.

The model isomorphism criterion therefore judges some theories to be inequivalent that we actually have good reason to consider equivalent. One is therefore not licensed to conclude that Hamiltonian and Lagrangian mechanics are inequivalent theories simply because their models are not isomorphic. After all, they could be equivalent in precisely the same sense as these two formulations of general relativity. If we want to better understand the relationship between Hamiltonian and Lagrangian mechanics, we need a better standard for equivalence of theories than the model isomorphism criterion.

One particularly influential criterion for equivalence, called definitional equivalence, was introduced into philosophy of science by Glymour $(1971,1977,1980)$. The basic idea behind Glymour's proposal is simple: Theories $T_{1}$ and $T_{2}$ are definitionally equivalent if $T_{1}$ can define all of the vocabulary that $T_{2}$ uses, and in a compatible way, $T_{2}$ can define all of the vocabulary that $T_{1}$ uses. Definitionally equivalent theories intuitively "say the same thing," but just use different vocabulary to say it. Indeed, one can show that there is a precise sense in which in which definitionally equivalent theories are intertranslatable (Barrett and Halvorson, 2016b, Theorems 1 and 2). Although definitional equivalence is often used by logicians as the standard for equivalence of theories, we will not do so here.

There are two reasons for this. ${ }^{10}$ First, like the model isomorphism criterion, definitional equivalence is too strict as a standard for equivalence. There are theories that are not definitionally equivalent that we nonetheless have good reason to consider equivalent. Consider the following two examples.

Example 2. It is well-known that there are many different ways to formulate Euclidean geometry. For example, it can be formulated using the apparatus of "lines" or it can be formulated using the apparatus of "points."

\footnotetext{
${ }^{9}$ This claim of equivalence depends subtly on what we mean by "general relativity," and more precisely, on what we include as allowable forms of matter fields on spacetime. For example, spinor fields are more easily represented in the framework of $(3,1)$ general relativity (Wald, 1984, Ch. 13). One might take this to be an important difference between the two formulations, and use it to judge them inequivalent. I take this to be further evidence for my Payoff 1 in what follows. Thanks to Erik Curiel for pointing me to this issue.

${ }^{10}$ See also the discussion in Weatherall (2016a, §5).
} 


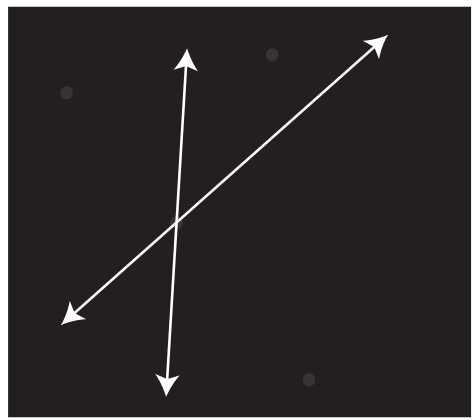

Euclidean geometry with lines

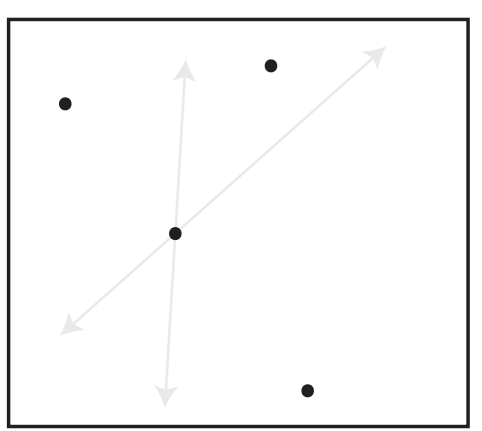

Euclidean geometry with points

Although one might like to consider these two formulations to be equivalent after all, they are both standardly referred to as "Euclidean geometry" - one can easily show that they are not definitionally equivalent. ${ }^{11}$

Example 3. General relativity is normally formulated geometrically by using a smooth manifold with various structures on it. But in the early 1970s, Geroch (1972) noticed that general relativity could also be formulated in a purely algebraic fashion by using a structure called an "Einstein algebra." One can think of the elements of an Einstein algebra as the smooth scalar functions on a spacetime.

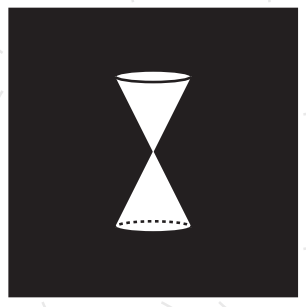

general relativity

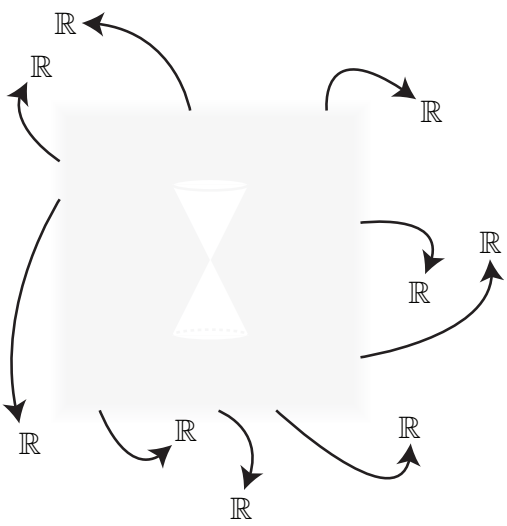

the theory of Einstein algebras

The theory of Einstein algebras was originally proposed by Geroch as another way to capture "the entire content of general relativity" (Geroch, 1972, p. 1); the theory is simply another "version" of general relativity. The structure of a relativistic spacetime can be recovered from that of an Einstein algebra, and

${ }^{11}$ See Barrett and Halvorson (2017a) for detailed discussion of this case. 
vice versa. One therefore might want to consider the theory of Einstein algebras equivalent to general relativity. Definitional equivalence, however, does not provide one with the means to do this. These two theories are not definitionally equivalent (Rosenstock, Barrett, and Weatherall, 2015). ${ }^{12}$

If one wants to capture the sense of equivalence in play in Examples 2 and 3 , one needs a standard for equivalence that is more liberal than definitional equivalence.

Example 3 also illustrates our second reason for not using definitional equivalence as our standard for equivalence: Definitional equivalence is a difficult criterion to apply to theories that are formulated outside the framework of firstorder logic. In order to say whether two theories are definitionally equivalent, one needs to be able to precisely state what "languages" the theories are formulated in and what counts as a "definition" in these languages. Although there is a standard way to handle both of these tasks when working with first-order theories, they become considerably more difficult when one is working with more general kinds of theories, like physical theories. ${ }^{13}$

One would like a standard of equivalence that does not suffer from the shortcomings of the model isomorphism criterion and definitional equivalence. Fortunately, such a standard exists, and it is called categorical equivalence. This criterion was first described by Eilenberg and Mac Lane $(1942,1945)$, but was only recently introduced into philosophy of science by Halvorson (2012, 2016) and Weatherall (2016a). ${ }^{14}$ Categorical equivalence is the standard for equivalence of theories that we will employ in this paper. It retains one of the basic intuitions behind definitional equivalence: Categorically equivalent theories are "intertranslatable." If $T_{1}$ and $T_{2}$ are categorically equivalent, then models of $T_{1}$ can be "translated" or "converted" into models of $T_{2}$, and vice versa, and these translations are (up to isomorphism) inverses of one another.

In order to precisely define categorical equivalence, we need to do a bit of work. We begin with the following simple observation: The class of models of a physical theory often has the structure of a category. We will call this category the category of models of the theory. Two theories are categorically

\footnotetext{
${ }^{12}$ Philosophers of physics have debated whether we should consider these theories equivalent. See Earman (1977a,b, 1979, 1986, 1989a,b), Rynasiewicz (1992), Bain (2003), and Rosenstock, Barrett, and Weatherall (2015) for discussion.

${ }^{13}$ Glymour proposes that we use the concept of "covariant definability" to say what counts as a definition outside the framework of first-order logic. This proposal, however, only works when one is dealing with theories formulated using the apparatus of differential geometry, so it does not apply to the theory of Einstein algebras. Of course, this proposal also does little to address the task of stating what "language" a physical theory is formulated in. Hudetz (2016) has recently proposed a standard of equivalence, called definable categorical equivalence, that allows one to handle these difficulties. I suspect that one will be able to prove results about definable categorical equivalence that correspond to Propositions 1, 2, and 3 in what follows, but that is left for future work.

${ }^{14}$ Halvorson $(2012,2016)$ and Weatherall (2016a, 2017, 2016b) describe various motivations behind categorical equivalence, while Barrett and Halvorson (2016b) show how it is related in logical strength to other criteria for equivalence that have been proposed. The reader is encouraged to consult Mac Lane (1971) and Borceux (1994) for details on category theory. In what follows, we take for granted the precise definitions of a category and a functor.
} 
equivalent if their categories of models are "isomorphic" in an appropriate sense. Describing this sense requires some basic preliminaries from category theory. A category $C$ is a collection of objects with arrows between the objects that satisfy some basic properties. The arrows in a category $C$ can be thought of as the "structure-preserving maps" between the objects of the category. An object in the category of models of a physical theory is just a model of the theory. The arrows between objects in the category of models encode the relationships that different models of the physical theory might bear to one another.

A functor $F: C \rightarrow D$ is a structure-preserving map between categories $C$ and $D$. One can think of a functor as a "way to translate" objects and arrows of $C$ into objects and arrows of $D$. A functor $F: C \rightarrow D$ is full if for all objects $c_{1}, c_{2}$ in $C$ and arrows $g: F c_{1} \rightarrow F c_{2}$ in $D$ there exists an arrow $f: c_{1} \rightarrow c_{2}$ in $C$ such that $F f=g . F$ is faithful if $F f=F g$ implies that $f=g$ for all arrows $f: c_{1} \rightarrow c_{2}$ and $g: c_{1} \rightarrow c_{2}$ in $C$. $F$ is essentially surjective if for every object $d$ in $D$ there exists an object $c$ in $C$ such that $F c \cong d$. A functor $F: C \rightarrow D$ that is full, faithful, and essentially surjective is called an equivalence. The categories $C$ and $D$ are equivalent if there exists an equivalence between them.

Following Weatherall (2016a), we will employ the following standard for equivalence between physical theories.

Criterion. Theories $T_{1}$ and $T_{2}$ are categorically equivalent if there is an equivalence between the category of models of $T_{1}$ and the category of models of $T_{2}$ that preserves the empirical content of the theories.

Of course, we have yet to say what it might mean for a functor to "preserve empirical content." This requirement is simply meant to guarantee that $T_{1}$ and $T_{2}$ are empirically equivalent, in addition to being expressed in equivalent formal frameworks. Since different theories endow their models with empirical content in different ways, this requirement is sensitive to the theories being considered. When we consider two specific functors between Hamiltonian and Lagrangian mechanics in the following section, we will state a precise sense in which they "preserve empirical content."

Categorical equivalence does not suffer from the same shortcomings as the criteria considered above. It is a more liberal standard for equivalence than both the model isomorphism criterion and definitional equivalence: The pairs of theories from Examples 1, 2, and 3 are categorically equivalent.

Example 1: $(1,3)$ general relativity and $(3,1)$ general relativity are categorically equivalent. The most natural way to define categories of models for these theories is to take the objects of the categories to be manifolds with metrics of the appropriate signature, and to take the arrows in the categories to be isometries. One can then easily verify that these two categories are equivalent, and furthermore, that the functor $F$ realizing the equivalence preserves the empirical content of the theories. (The models $\left(M, g_{a b}\right)$ and $F\left(M, g_{a b}\right)$ agree about everything of physical significance: They have the same covariant derivative operator, they agree about which curve are timelike, spacelike, and lightlike, etc.) 
Example 2: The category of models of Euclidean geometry with points is equivalent to the category of models of Euclidean geometry with lines. This follows from the results proven by Barrett and Halvorson (2017a, 2016b).

Example 3: Categorical equivalence can also be used to capture the sense in which general relativity and the theory of Einstein algebras are equivalent theories (Rosenstock, Barrett, and Weatherall, 2015).

Examples 1 and 3 illustrate in addition that categorical equivalence can easily be applied outside the framework of first-order logic. We will therefore use it as our standard of equivalence in what follows.

\section{Preliminaries on classical mechanics}

Now that we have in hand a standard for equivalence of theories, we can consider the specific case of Hamiltonian and Lagrangian mechanics. We begin with some preliminaries on these two theories. ${ }^{15}$

Consider a classical system of $n$ particles. The positions of these particles can be encoded by a single point $p$ in a $3 n$-dimensional smooth manifold $M$ called configuration space. The statespaces of Hamiltonian and Lagrangian mechanics are "built on top of" configuration space.

\section{Hamiltonian mechanics}

The statespace of Hamiltonian mechanics is the $6 n$-dimensional smooth manifold $T^{*} M$, the cotangent bundle of configuration space. A point $(p, \omega) \in$ $T^{*} M$ encodes the positions and momenta of all the particles in the system. The cotangent bundle $T^{*} M$ comes naturally equipped with various geometric structures that are employed in Hamiltonian mechanics. For example, the canonical one-form is the smooth tensor field on $T^{*} M$ that takes the value

$$
\left.\theta_{a}\right|_{(p, \omega)}=\pi^{*}(\omega)
$$

at a point $(p, \omega) \in T^{*} M$, where $\pi^{*}$ is the pullback of the standard projection $\pi: T^{*} M \rightarrow M$. And the canonical symplectic form on $T^{*} M$ is the smooth field $\Omega_{a b}=-d_{a} \theta_{b}$. One can verify that $\Omega_{a b}$ is indeed a symplectic form (i.e. it is closed, anti-symmetric, and non-degenerate).

As with other mathematical objects, there are "structure-preserving maps" between cotangent bundles. Given a diffeomorphism $f: M_{1} \rightarrow M_{2}$, the point*transformation $T^{*} f: T^{*} M_{2} \rightarrow T^{*} M_{1}$ is the map defined by $T^{*} f:(p, \omega) \mapsto$ $\left(f^{-1}(p), f^{*}(\omega)\right)$. Point*-transformations are diffeomorphisms and also preserve

\footnotetext{
${ }^{15}$ The reader is encouraged to consult Abraham and Marsden (1978) or Mac Lane (1968) for further details. We will use the "abstract index notation" for tensor fields on the tangent and cotangent bundles $T_{*} M$ and $T^{*} M$. We will also occasionally use this notation for tensors on $M$, but it will always be clear from context which is meant.
} 
the geometric structures on the cotangent bundle. Indeed, if $\theta_{a}$ and $\widehat{\theta}_{a}$ are the canonical one-forms on $T^{*} M_{1}$ and $T^{*} M_{2}$, then every point*-transformation $T^{*} f: T^{*} M_{2} \rightarrow T^{*} M_{1}$ satisfies $\left(T^{*} f\right)^{*}\left(\theta_{a}\right)=\widehat{\theta}_{a}$. This immediately implies in addition that $\left(T^{*} f\right)^{*}\left(\Omega_{a b}\right)=\widehat{\Omega}_{a b}$, where $\Omega_{a b}$ and $\widehat{\Omega}_{a b}$ are the canonical symplectic forms on $T^{*} M_{1}$ and $T^{*} M_{2}$.

The structure of the cotangent bundle alone, however, is not enough to run the theory. One also needs to provide some energy properties in order to say how the system will evolve over time. In Hamiltonian mechanics this is done by specifying a Hamiltonian for the system, a smooth scalar function $H: T^{*} M \rightarrow$ $\mathbb{R}$ that encodes the "total energy" of the system. A model of Hamiltonian mechanics is then a pair $\left(T^{*} M, H\right)$, where $T^{*} M$ is a cotangent bundle and $H$ is a Hamiltonian. The Hamiltonian vector field $\left(X_{H}\right)^{a}$ is the unique smooth field on $T^{*} M$ that satisfies $\Omega_{a b}\left(X_{H}\right)^{a}=d_{b} H$. The non-degeneracy of $\Omega_{a b}$ guarantees that the field $\left(X_{H}\right)^{a}$ exists and is unique. Given Hamiltonians $H_{1}: T^{*} M \rightarrow \mathbb{R}$ and $H_{2}: T^{*} M \rightarrow \mathbb{R}$, one can easily verify that $\left(X_{H_{1}}\right)^{a}=\left(X_{H_{2}}\right)^{a}$ if and only if $H_{1}=H_{2}+c$ for some constant $c \in \mathbb{R}$. The Hamiltonian vector field encodes the dynamics of the system. If the system's initial state is given by the point $(p, \omega) \in T^{*} M$, then the system evolves by "flowing" this state along the Hamiltonian vector field. More precisely, the integral curves of the vector field $\left(X_{H}\right)^{a}$ are the trajectories through the Hamiltonian statespace $T^{*} M$ along which the system might evolve, and one proves that Hamilton's equations hold along these curves (Abraham and Marsden, 1978, Proposition 3.3.2).

For our purposes, we will be particularly interested in "hyperregular" models of Hamiltonian mechanics. We take a moment here to say what this means. Given a model of Hamiltonian mechanics $\left(T^{*} M, H\right)$, the fiber derivative of $H$ is the map

$$
\mathcal{H}_{H}: T^{*} M \rightarrow T_{*} M \quad \mathcal{H}_{H}:(p, \alpha) \longmapsto\left(p, \mathcal{H}_{H}^{q}(\alpha)\right),
$$

where $T_{*} M$ is the tangent bundle of $M$ and the vector $\mathcal{H}_{H}^{p}(\alpha) \in T_{p} M$ is defined by its action

$$
\mathcal{H}_{H}^{p}(\alpha) \cdot \beta=\left.\frac{d}{d t} H(p, \alpha+t \beta)\right|_{t=0}
$$

on covectors $\beta \in T^{p} M$. The map $\mathcal{H}_{H}$ is smooth and fiber-preserving. When $\mathcal{H}_{H}$ is a diffeomorphism, the model $\left(T^{*} M, H\right)$ is called hyperregular and the Hamiltonian $H$ is referred to as a hyperregular Hamiltonian.

\section{Lagrangian mechanics}

Lagrangian mechanics is formulated in a similar manner. The statespace of Lagrangian mechanics is the $6 n$-dimensional tangent bundle $T_{*} M$ of configuration space. A point $(p, v) \in T_{*} M$ encodes the positions and velocities of all the particles in the system. The structure-preserving maps between tangent bundles are defined analogously to the above point*-transformations. Given a dif-

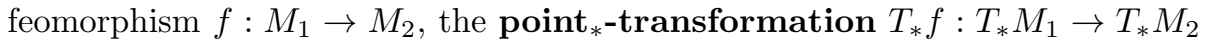
is defined by $T_{*} f:(p, v) \mapsto\left(f(p), f_{*}(v)\right)$. 
In Lagrangian mechanics, the energy properties of the system are encoded by the system's Lagrangian, a smooth scalar function $L: T_{*} M \rightarrow \mathbb{R}$ that specifies the "liveliness" or "activity" of the system. A model of Lagrangian mechanics is then a pair $\left(T_{*} M, L\right)$, where $T_{*} M$ is a tangent bundle and $L$ is a Lagrangian.

We will again be primarily interested in hyperregular models of Lagrangian mechanics. This concept is defined analogously to above. Given a model of Lagrangian mechanics $\left(T_{*} M, L\right)$, the fiber derivative of $L$ is the map

$$
\mathcal{L}_{L}: T_{*} M \rightarrow T^{*} M \quad \mathcal{L}_{L}:(p, v) \longmapsto\left(p, \mathcal{L}_{L}^{p}(v)\right),
$$

where the covector $\mathcal{L}_{L}^{p}(v) \in T^{p} M$ is defined by its action

$$
\mathcal{L}_{L}^{p}(v) \cdot w=\left.\frac{d}{d t} L(p, v+t w)\right|_{t=0}
$$

on vectors $w \in T_{p} M$. The map $\mathcal{L}_{L}$ is smooth and fiber-preserving, and it is often called the Legendre transformation associated with the Lagrangian $L$. When $\mathcal{L}_{L}$ is a diffeomorphism, the model $\left(T_{*} M, L\right)$ is called hyperregular and the Lagrangian $L$ is referred to as a hyperregular Lagrangian.

The total energy associated with the Lagrangian $L$ is the smooth scalar function $E_{L}: T_{*} M \rightarrow \mathbb{R}$ defined by $E_{L}(p, v)=\mathcal{L}_{L}^{p}(v) \cdot v-L(p, v)$. When $\left(T_{*} M, L\right)$ is hyperregular, the geometric structures from the statespace of Hamiltonian mechanics naturally transfer onto the statespace of Lagrangian mechanics. In particular, the tangent bundle $T_{*} M$ inherits a symplectic form $\left(\mathcal{L}_{L}\right)^{*}\left(\Omega_{a b}\right)$, where $\Omega_{a b}$ is the canonical symplectic form on $T^{*} M$. The tensor $\left(\mathcal{L}_{L}\right)^{*}\left(\Omega_{a b}\right)$ is guaranteed to be a symplectic form because $\mathcal{L}_{L}$ is a diffeomorphism. The Lagrangian vector field $\left(X_{L}\right)^{a}$ on $T_{*} M$ is the unique smooth field on $T_{*} M$ that satisfies $\left(\mathcal{L}_{L}\right)^{*}\left(\Omega_{a b}\right)\left(X_{L}\right)^{a}=d_{b} E_{L}$. Like the Hamiltonian vector field, the Lagrangian vector field encodes the dynamics of the system. The integral curves of $\left(X_{L}\right)^{a}$ are the trajectories through the Lagrangian statespace $T_{*} M$ along which the system evolves, and one accordingly proves that the Euler-Lagrange equations hold along these curves (Abraham and Marsden, 1978, Theorem 3.5.17).

\section{Are Hamiltonian and Lagrangian mechanics equivalent theories?}

In order to say whether or not Hamiltonian and Lagrangian mechanics are categorically equivalent, we need to complete two tasks. We will first describe "translations" between the theories that are candidates for functors, and then we will define categories of models for these two theories.

As we have seen, Hamiltonian mechanics is formulated using the mathematical apparatus of cotangent bundles and Hamiltonians, while Lagrangian mechanics is formulated using the mathematical apparatus of tangent bundles and Lagrangians. But there is a natural way to translate between these two formulations. One can convert hyperregular models of Lagrangian mechanics 
into hyperregular models of Hamiltonian mechanics, and in addition, structurepreserving maps between tangent bundles into structure-preserving maps between cotangent bundles. ${ }^{16}$ We define this "translation" $F$ as follows.

$$
\begin{aligned}
& F:\left(T_{*} M, L\right) \longmapsto\left(T^{*} M, E_{L} \circ \mathcal{L}_{L}^{-1}\right) \\
& F: T_{*} f \longmapsto T^{*}\left(f^{-1}\right)
\end{aligned}
$$

The model $\left(T^{*} M, E_{L} \circ \mathcal{L}_{L}^{-1}\right)$ of Hamiltonian mechanics is hyperregular. Indeed, one can show that $\mathcal{H}_{E_{L} \circ \mathcal{L}_{L}^{-1}}=\mathcal{L}_{L}^{-1}$ (Abraham and Marsden, 1978, Proposition 3.6.8).

There is also a natural way to convert hyperregular models of Hamiltonian mechanics into hyperregular models of Lagrangian mechanics, and structurepreserving maps between cotangent bundles into structure-preserving maps between tangent bundles. We define this "translation" $G$ as follows.

$$
\begin{aligned}
& G:\left(T^{*} M, H\right) \longmapsto\left(T_{*} M,\left(\theta_{a}\left(X_{H}\right)^{a}-H\right) \circ \mathcal{H}_{H}^{-1}\right) \\
& G: T^{*} f \longmapsto T_{*}\left(f^{-1}\right)
\end{aligned}
$$

The field $\theta_{a}$ is the canonical one-form on $T^{*} M$ and $\left(X_{H}\right)^{a}$ is the Hamiltonian vector field on $T^{*} M$ corresponding to $H$. One can verify that the model $\left(T_{*} M,\left(\theta_{a}\left(X_{H}\right)^{a}-H\right) \circ \mathcal{H}_{H}^{-1}\right)$ is a hyperregular model of Lagrangian mechanics with $\mathcal{L}_{\left(\theta_{a}\left(X_{H}\right)^{a}-H\right) \circ \mathcal{H}_{H}^{-1}}=\mathcal{H}_{H}^{-1}$ (Abraham and Marsden, 1978, Proposition 3.6.7).

We should take a moment to unravel the idea behind the translations $F$ and $G$. Given a hyperregular model of Lagrangian mechanics $\left(T_{*} M, L\right), F$ builds a corresponding model of Hamiltonian mechanics. It does this by using the Legendre transformation $\mathcal{L}_{L}$ to "pull back" the total energy function $E_{L}$ onto the cotangent bundle $T^{*} M$. And $F$ simply converts the point ${ }_{*}$-transformation $T_{*} f$ into the corresponding point*-transformation $T^{*}\left(f^{-1}\right)$. The idea behind $G$ is perfectly analogous. Given a hyperregular model of Hamiltonian mechanics $\left(T^{*} M, H\right), G$ builds a corresponding model of Lagrangian mechanics. The scalar function $\theta_{a}\left(X_{H}\right)^{a}-H$ on the cotangent bundle $T^{*} M$ encodes the "liveliness" or "activity" of the Hamiltonian system. $G$ uses the fiber derivative $\mathcal{H}_{H}$ to "pull back" this function onto $T_{*} M$. And $G$ converts the point*-transformation $T^{*} f$ into the corresponding point ${ }_{*}$-transformation $T_{*}\left(f^{-1}\right)$.

The translations $F$ and $G$ are closely related to one another. Indeed, we have the following fundamental result (Abraham and Marsden, 1978, Theorem 3.6.9).

\footnotetext{
${ }^{16}$ For the purposes of this paper we will only consider the case of hyperregular Hamiltonian and Lagrangian mechanics. One can, of course, consider the more general case, but I conjecture that there the theories will be inequivalent according to any reasonable standard of equivalence. There is no natural way to translate a non-hyperregular model of the one theory into a model of the other. In the hyperregular case, the maps $F$ and $G$ defined below are the only natural candidates for "good translations" between Hamiltonian and Lagrangian mechanics. There are no other known maps that preserve the empirical content of the theories in the way made precise by Theorems 2 and 3.
} 
Theorem 1. $F$ and $G$ are "inverses," in the sense that $G F\left(T_{*} M, L\right)=\left(T_{*} M, L\right)$ and $F G\left(T^{*} M, H\right)=\left(T^{*} M, H\right)$ for all hyperregular models of Lagrangian mechanics $\left(T_{*} M, L\right)$ and hyperregular models of Hamiltonian mechanics $\left(T^{*} M, H\right)$.

Theorem 1 strongly suggests that we will be able to show that $F$ and $G$ are equivalences between Hamiltonian and Lagrangian mechanics. But in order to apply categorical equivalence as it is stated above, we will need to verify in addition that there is a sense in which $F$ and $G$ "preserve the empirical content" of Hamiltonian and Lagrangian mechanics. The following two theorems are normally taken to do precisely this. These results guarantee that $F$ and $G$ both map models of the one theory to models of the other theory that agree on the allowable trajectories for particles in the system.

One more piece of terminology is necessary in order to state these results. If $\gamma: I \rightarrow T_{*} M$ is an integral curve of $\left(X_{L}\right)^{a}$, then we call the curve $\pi \circ \gamma: I \rightarrow M$ a base integral curve of $\left(X_{L}\right)^{a}$, where $\pi: T_{*} M \rightarrow M$ is the canonical projection. A base integral curve of $\left(X_{L}\right)^{a}$ encodes one way that the positions of particles in a system with Lagrangian $L$ might evolve over time. The base integral curves of $\left(X_{H}\right)^{a}$ are defined analogously, and they encode the ways that the positions of particles in a system with Hamiltonian $H$ might evolve over time. The following theorem captures a sense in which $F$ preserves empirical content (Abraham and Marsden, 1978, Theorem 3.6.2).

Theorem 2. If $\left(T_{*} M, L\right)$ is a hyperregular model of Lagrangian mechanics and $F\left(T_{*} M, L\right)=\left(T^{*} M, E_{L} \circ \mathcal{L}_{L}^{-1}\right)$ is the corresponding model of Hamiltonian mechanics, then $\left(X_{L}\right)^{a}$ and $\left(X_{E_{L} \circ \mathcal{L}_{L}^{-1}}\right)^{a}$ have the same base integral curves.

The model $\left(T_{*} M, L\right)$ of Lagrangian mechanics and the model $F\left(T_{*} M, L\right)$ of Hamiltonian mechanics therefore agree on how the positions of particles in the system will evolve over time. Theorem 3 follows immediately from Theorems 1 and 2. It guarantees that the models $\left(T^{*} M, H\right)$ and $G\left(T^{*} M, H\right)$ also agree on how the positions of particles will evolve over time.

Theorem 3. If $\left(T^{*} M, H\right)$ is a hyperregular model of Hamiltonian mechanics and $G\left(T^{*} M, H\right)=\left(T_{*} M,\left(\theta_{a}\left(X_{H}\right)^{a}-H\right) \circ \mathcal{H}_{H}^{-1}\right)$ is the corresponding model of Lagrangian mechanics, then $\left(X_{H}\right)^{a}$ and $\left(X_{\left(\theta_{b}\left(X_{H}\right)^{b}-H\right) \circ \mathcal{H}_{H}^{-1}}\right)^{a}$ have the same base integral curves.

Theorems 2 and 3 together capture a robust sense in which the translations $F$ and $G$ preserve empirical content.

In order to say whether or not $F$ or $G$ can be thought of as equivalences between Hamiltonian and Lagrangian mechanics, we need to define categories of models for these theories. In general, there is more than one way to define a category of models for a physical theory. Different ways of understanding the mathematical apparatus behind the theory will correspond to different ways of defining a category of models for the theory. In particular, the way that one defines the arrows in the category of models depends on which mathematical 
structures one takes to be essential in formulating the theory. ${ }^{17}$ In what follows, therefore, we consider three different ways of defining categories of models for Hamiltonian and Lagrangian mechanics. In each case we prove whether or not $F$ and $G$ are equivalences.

\section{Tangent bundle vs. cotangent bundle}

The first way of defining categories of models for Hamiltonian and Lagrangian mechanics that we will consider substantiates the standard view about these theories. We define the categories of models $\mathbf{L a g}_{1}$ and $\mathbf{H a m}_{1}$ for Lagrangian and Hamiltonian mechanics as follows.

- An object in the category $\mathbf{L a g}_{1}$ is a hyperregular model $\left(T_{*} M, L\right)$ of Lagrangian mechanics. An arrow $\left(T_{*} M_{1}, L_{1}\right) \rightarrow\left(T_{*} M_{2}, L_{2}\right)$ is a point $*^{-}$ transformation $T_{*} f: T_{*} M_{1} \rightarrow T_{*} M_{2}$ that preserves the Lagrangian in the sense that $L_{2} \circ T_{*} f=L_{1}$.

- An object in the category $\mathbf{H a m}_{1}$ is a hyperregular model $\left(T_{*} M, H\right)$ of Hamiltonian mechanics. An arrow $\left(T^{*} M_{1}, H_{1}\right) \rightarrow\left(T^{*} M_{2}, H_{2}\right)$ is a point*transformation $T^{*} f: T^{*} M_{1} \rightarrow T^{*} M_{2}$ that preserves the Hamiltonian in the sense that $H_{2} \circ T^{*} f=H_{1}$.

One can easily verify that $\mathbf{L a g}_{1}$ and $\mathbf{H a m}_{1}$ are categories. This is one particularly natural way of defining categories of models for Hamiltonian and Lagrangian mechanics. Arrows in $\mathbf{L a g}_{1}$ are simply required to preserve the structure of the tangent bundle and the Lagrangian, while arrows in $\mathbf{H a m}_{1}$ are required to preserve the structure of the cotangent bundle and the Hamiltonian.

We now have the following simple result.

Proposition 1. F: $\boldsymbol{L a g}_{1} \rightarrow \boldsymbol{H a m}_{1}$ and $G: \boldsymbol{H a m}_{1} \rightarrow \boldsymbol{L a g}_{1}$ are equivalences.

Proof. See appendix.

Proposition 1 captures a sense in which the standard view about Hamiltonian and Lagrangian mechanics is correct. According to one particularly natural way of defining categories of models for these theories, they are categorically equivalent. Given a hyperregular model of Lagrangian mechanics one can use the map $F$ to construct a hyperregular model of Hamiltonian mechanics that has the same empirical content. And conversely, given a hyperregular model of Hamiltonian mechanics one can use the map $G$ to construct a hyperregular model of Lagrangian mechanics that has the same empirical content. Proposition 1 therefore provides us with a clear and precise sense in which Hamiltonian and Lagrangian mechanics are equivalent theories.

\footnotetext{
${ }^{17}$ Weatherall (2017) presents examples of categories of models for other physical theories. See Curiel (2014) for a nice discussion of which mathematical structures in Lagrangian and Hamiltonian mechanics one should take to have physical significance.
} 


\section{Tangent bundle vs. symplectic manifold}

But there are other ways to define categories of models for Hamiltonian and Lagrangian mechanics. Indeed, we will now consider one alternative way to define a category of models for Hamiltonian mechanics.

The motivation behind this alternative way of defining a category of models for Hamiltonian mechanics is the following: Hamiltonian mechanics can be formulated on an arbitrary symplectic manifold, rather than on a cotangent bundle. ${ }^{18}$ The basic idea is that the cotangent bundle has strictly "more structure" than is necessary to stipulate the dynamics of Hamiltonian mechanics. In fact, as one can verify by examining the definition of the Hamiltonian vector field above, the only structures that are necessary are a symplectic form and a Hamiltonian. Since the cotangent bundle has "superfluous structure," mathematicians and physicists will often formulate Hamiltonian mechanics on an arbitrary symplectic manifold instead. A model of this more general formulation of Hamiltonian mechanics is a tuple $\left(M, \Omega_{a b}, H\right)$, where $\Omega_{a b}$ is a symplectic form on the manifold $M$ and $H: M \rightarrow \mathbb{R}$ is a smooth scalar function, again called the Hamiltonian. One again defines the Hamiltonian vector field to be the unique field satisfying $\Omega_{a b}\left(X_{H}\right)^{a}=d_{b} H$, and then shows that Hamilton's equations hold along integral curves of $\left(X_{H}\right)^{a}$.

If one has in mind this more general formulation of Hamiltonian mechanics, then one will naturally be led to consider the following category of models $\mathbf{H a m}_{2}$ for Hamiltonian mechanics instead of $\mathbf{H a m}_{1}$.

- An object in the category $\mathbf{H a m}_{2}$ is a tuple $\left(M, \Omega_{a b}, H\right)$, as defined above. An arrow between objects $\left(M, \Omega_{a b}, H\right) \rightarrow\left(N, \widehat{\Omega}_{a b}, \widehat{H}\right)$ in $\mathbf{H a m}_{2}$ is a diffeomorphism $f: M \rightarrow N$ that preserves the symplectic form and the Hamiltonian, in the sense that $f^{*}\left(\widehat{\Omega}_{a b}\right)=\Omega_{a b}$ and $\widehat{H} \circ f=H$.

One can easily verify that $\mathbf{H a m}_{2}$ is a category.

In order for $F$ to be a candidate for a functor from $\mathbf{L a g}_{1}$ to $\mathbf{H a m}_{2}$, we need to slightly revise the earlier definition. For every object $\left(T_{*} M, L\right)$ in $\operatorname{Lag}_{1}$ we need $F\left(T_{*} M, L\right)$ to be an object in $\mathbf{H a m}_{2}$, so we need to think of the map $F$ as follows:

$$
\begin{aligned}
& F:\left(T_{*} M, L\right) \longmapsto\left(T^{*} M, \Omega_{a b}, E_{L} \circ \mathcal{L}_{L}^{-1}\right) \\
& F: T_{*} f \longmapsto T^{*}\left(f^{-1}\right)
\end{aligned}
$$

where $\Omega_{a b}$ is the canonical symplectic form on $T^{*} M$. While $F$ is a functor from $\mathbf{L a g}_{1}$ to $\mathbf{H a m}_{2}$ (see Lemma 2 in the appendix), note that $G$ is not a functor from $\mathbf{H a m}_{2}$ to $\mathbf{L a g}_{1}$. There simply is no way to think of $G$ as a "translation" from this general formulation of Hamiltonian mechanics to Lagrangian mechanics. The map $G$ is only defined for models of Hamiltonian mechanics whose

\footnotetext{
${ }^{18}$ See Abraham and Marsden $(1978, \S 3.3)$ for details on this formulation. It is considered by North (2009) and leads her to conclude that models of Hamiltonian mechanics have less structure than models of Lagrangian mechanics. One wonders whether there is also a correspondingly general formulation of Lagrangian mechanics.
} 
underlying manifold is a cotangent bundle. Since not every symplectic manifold is a cotangent bundle, there are objects of $\mathbf{H a m}_{2}$ on which $G$ is not defined.

This immediately implies that $G$ is not an equivalence. The following proposition shows that $F$ is not an equivalence either.

Proposition 2. Neither $F: \mathbf{L a g}_{1} \rightarrow \mathbf{H a m}_{2}$ nor $G: \mathbf{H a m}_{2} \rightarrow \mathbf{L a g}_{1}$ is an equivalence.

Proof. See appendix.

Proposition 2 captures a sense in which Lagrangian mechanics is inequivalent to this general formulation of Hamiltonian mechanics. This verdict is intuitive. The mathematical apparatus of Lagrangian mechanics simply is not the same as the mathematical apparatus of this general formulation of Hamiltonian mechanics. Objects in $\mathbf{L a g}_{1}$ have "bundle structure"; there is a privileged way in which tangent bundles split into "base points" and "fibers" above these base points. Since objects in $\mathbf{H a m}_{2}$ are arbitrary symplectic manifolds, and not necessarily cotangent bundles, they do not have this structure. This captures the basic idea that North (2009) has about the relationship between these two theories: Models of this more general formulation of Hamiltonian mechanics have "less structure" than models of Lagrangian mechanics. Since the functor $F: \mathbf{L a g}_{1} \rightarrow \mathbf{H a m}_{2}$ is not an equivalence, this provides us with a good reason to consider Lagrangian mechanics inequivalent to Hamiltonian mechanics formulated on an arbitrary symplectic manifold.

\section{Lagrangian vector field vs. Hamiltonian vector field}

The first case that we considered captured a sense in which Hamiltonian and Lagrangian mechanics might be considered equivalent, while the second case captured a sense in which Hamiltonian mechanics - if it is understood as formulated on an arbitrary symplectic manifold - can be considered inequivalent to Lagrangian mechanics. This final case demonstrates another sense in which one might consider these two theories inequivalent.

This sense corresponds to another natural way of defining categories of models for Lagrangian and Hamiltonian mechanics. The arrows in the categories $\mathbf{L a g}_{1}, \mathbf{H a m}_{1}$, and $\mathbf{H a m}_{2}$ were required to preserve the Lagrangian and Hamiltonian functions "on the dot." One might worry that this is too strict a requirement. One might think, for example, that the Hamiltonian and Lagrangian vector fields encode all of the dynamically important information about these two theories. The exact real number value that the Hamiltonians and Lagrangians take is not all that important; it is a kind of "surplus structure" to the theory. The following example makes this worry precise. The worry is illustrated here by using models of Hamiltonian mechanics, but one could just as easily use models of Lagrangian mechanics.

Example 4. Let $M$ be a smooth manifold with $g_{a b}$ a Riemannian metric on $M$ and $c \in \mathbb{R}$ a positive real number. Consider the Hamiltonians $H_{1}: T^{*} M \rightarrow \mathbb{R}$ 
and $H_{2}: T^{*} M \rightarrow \mathbb{R}$ defined by

$$
H_{1}:\left(p, \omega_{a}\right) \longmapsto g^{a b} \omega_{a} \omega_{b} \quad H_{2}:\left(p, \omega_{a}\right) \longmapsto g^{a b} \omega_{a} \omega_{b}+c
$$

One can easily verify that $\left(T^{*} M, H_{1}\right)$ and $\left(T^{*} M, H_{2}\right)$ are hyperregular models of Hamiltonian mechanics. Since $H_{1}$ and $H_{2}$ differ only by a constant, we see that $\left(X_{H_{1}}\right)^{a}=\left(X_{H_{2}}\right)^{a}$. One might therefore claim that these two models of Hamiltonian mechanics encode precisely the same information about a physical system. They agree about how the positions and momenta of the particles in the system will evolve over time. The two models are, however, not isomorphic in the category $\mathbf{H a m}_{1}$ nor in the category $\mathbf{H a m}_{2}$. In fact, one can show there are no arrows between these objects in $\mathbf{H a m}_{1}$ and $\mathbf{H a m}_{2}$. This follows from the fact that $H_{1}(p, \mathbf{0})=0$ for every $p \in M$, while $H_{2}$ is positive everywhere on $T^{*} M$.

If one thinks that the two models from Example 4 encode the same information about the system under consideration, then one will want the category of models for Hamiltonian mechanics to reflect this fact. This thought leads one to define new categories of models $\mathbf{H a m}_{3}$ and $\mathbf{L a g}_{2}$ for Hamiltonian and Lagrangian mechanics. In these new categories, the arrows are not required to preserve the Hamiltonian and Lagrangian functions "on the dot." Rather, they are merely required to preserve the Hamiltonian and Lagrangian vector fields.

- An object in the category $\mathbf{L a g}_{2}$ is a hyperregular model $\left(T_{*} M, L\right)$ of Lagrangian mechanics. An arrow $\left(T_{*} M_{1}, L_{1}\right) \rightarrow\left(T_{*} M_{2}, L_{2}\right)$ is a point $*^{-}$ transformation $T_{*} f: T_{*} M_{1} \rightarrow T_{*} M_{2}$ that preserves the Lagrangian vector fields in the sense that $\left(T_{*} f\right)_{*}\left(X_{L_{1}}\right)^{a}=\left(X_{L_{2}}\right)^{a}$.

- An object in the category $\mathbf{H a m}_{3}$ is a hyperregular model $\left(T^{*} M, H\right)$ of Hamiltonian mechanics. An arrow $\left(T^{*} M_{1}, H_{1}\right) \rightarrow\left(T^{*} M_{2}, H_{2}\right)$ is a point*transformation $T^{*} f: T_{*} M_{1} \rightarrow T_{*} M_{2}$ that preserves the Hamiltonian vector fields in the sense that $\left(T^{*} f\right)_{*}\left(X_{H_{1}}\right)^{a}=\left(X_{H_{2}}\right)^{a}$.

As before, one can easily verify that these are categories. Arrows in $\mathbf{L a g}_{2}$ preserve the structure of the tangent bundle and the Lagrangian vector field; arrows in $\mathbf{H a m}_{3}$ preserve the structure of the cotangent bundle and the Hamiltonian vector field. One can easily verify that the two models from Example 4 are isomorphic as objects in $\mathbf{H a m}_{3}$.

We can now ask whether or not $F$ and $G$ are equivalences between $\mathbf{L a g}_{2}$ and $\mathbf{H a m}_{3}$. The following proposition answers this question in the negative.

Proposition 3. Neither F: $\mathbf{L a g}_{2} \rightarrow \mathbf{H a m}_{3}$ nor $G: \mathbf{H a m}_{3} \rightarrow \mathbf{L a g}_{2}$ is an equivalence.

Proof. See appendix. ${ }^{19}$

\footnotetext{
${ }^{19}$ The proof of Proposition 3 shows that there are Lagrangians that give rise to the same Lagrangian vector field, but do not "translate" into Hamiltonians that give rise to the same Hamiltonian vector field. I take this to substantiate Curiel's remark that the "Legendre transform does not respect the kinematical constraints of Lagrangian mechanics" (Curiel, 2014, 42). The seeds for the proof of Proposition 3 are contained in (Abraham and Marsden, $1978,216-217)$.
} 
Proposition 2 substantiates the thought that North (2009) has about the inequivalence of these two formulations of classical mechanics. I take Proposition 6.3 to substantiate the thought that Curiel (2014) has about their inequivalence. Curiel remarks that the "Legendre transform does not respect the kinematical constraints of Lagrangian mechanics" (Curiel, 2014, 42). The proof of Proposition 6.3 essentially demonstrates this. It shows that there are Lagrangians that give rise to the same Lagrangian vector field, but do not translate - via the Legendre transform - into Hamiltonians that give rise to the same Hamiltonian vector field.

This proposition therefore captures another sense in which one might consider Hamiltonian and Lagrangian mechanics inequivalent theories. We should take a moment to unravel the intuition behind this verdict. There is a precise sense in which a Hamiltonian vector field $\left(X_{H}\right)^{a}$ encodes more information about the Hamiltonian $H$ than a Lagrangian vector field $\left(X_{L}\right)^{a}$ encodes about the Lagrangian $L$ or the total energy function $E_{L}$. The Hamiltonian vector field $\left(X_{H}\right)^{a}$ implicitly defines the Hamiltonian $H$ up to a constant. We have already remarked above that for Hamiltonians $H_{1}: T^{*} M \rightarrow \mathbb{R}$ and $H_{2}: T^{*} M \rightarrow \mathbb{R}$, $\left(X_{H_{1}}\right)^{a}=\left(X_{H_{2}}\right)^{a}$ if and only if $H_{1}=H_{2}+c$ for some constant $c \in \mathbb{R}$. If two models of Hamiltonian mechanics agree on the Hamiltonian vector field, then they must also agree (at least up to a constant) on the Hamiltonian.

The same cannot be said of models of Lagrangian mechanics. The Lagrangian vector field $\left(X_{L}\right)^{a}$ neither implicitly defines the Lagrangian $L$ nor the total energy function $E_{L}$ up to a constant. The proof of Proposition 3 demonstrates precisely this. It provides an example of two models of Lagrangian mechanics $\left(T_{*} M, L_{1}\right)$ and $\left(T_{*} M, L_{2}\right)$ that have the same Lagrangian vector field $\left(X_{L_{1}}\right)^{a}=\left(X_{L_{2}}\right)^{a}$, but do not agree (even up to a constant) on the Lagrangian nor on the total energy function of the system. The Hamiltonian vector field defines a scalar function up to a constant, while the Lagrangian vector field does not. The Hamiltonian vector field therefore gives rise to "more structure" than the Lagrangian vector field does. Indeed, one might summarize the content of Proposition 3 as follows: The mathematical apparatus of cotangent bundle plus Hamiltonian vector field is not the same as the mathematical apparatus of tangent bundle plus Lagrangian vector field. If one thinks of Hamiltonian and Lagrangian mechanics as best represented by the categories of models $\mathbf{H a m}_{3}$ and $\mathbf{L a g}_{2}$, then one has reason to consider these two theories inequivalent.

\section{Conclusion}

In his 1966 Nobel acceptance speech, Feynman (1966, p. 708) suggested that it is often useful to have different perspectives on a particular physical theory:

A good theoretical physicist today might find it useful to have a wide range of physical viewpoints and mathematical expressions of the same theory available. ${ }^{20}$

${ }^{20}$ Thanks to Jeremy Butterfield for pointing me toward this quote and to Alex Meehan for 
Having different viewpoints on a theory may help catalyze progress to newer and better theories. But in order to reap these benefits we first have to recognize when two viewpoints are providing us with views of the same theory, and when they are instead providing us with views of different theories. We have taken a step here towards doing precisely this in the simple case of classical mechanics. Proposition 1 substantiates the standard view about these theories and captures a clear sense in which one might consider them to be equivalent. But Propositions 2 and 3 show that categorical equivalence leaves room for one to follow North and Curiel and consider Hamiltonian and Lagrangian mechanics to be inequivalent theories. It all depends on how one understands the mathematical apparatus behind the two theories.

Given this end result, one might worry that we have not yet settled whether or not Hamiltonian and Lagrangian mechanics are equivalent theories. One might be disappointed by the "it depends" kind of answer that we have provided here. I do not think that we should be disappointed by this kind of answer. In fact, I believe that the answer provided here is the best kind of answer that we can expect when approaching questions of equivalence between theories. It takes a moment to explain why this is the case.

When we are presented with a question like "Are theories $T_{1}$ and $T_{2}$ equivalent?", the first order of business is to clarify what this relationship of equivalence amounts to. That is, we must begin by endorsing a particular standard of equivalence. Depending on the standard of equivalence that we endorse, we may find that there is some ambiguity about what exactly the theories $T_{1}$ and $T_{2}$ are. There may be a number of theories that are distinct according to our preferred standard of equivalence that nonetheless are standardly referred to by the name " $T_{1}$ " or " $T_{2}$." This is precisely what happened in the case of Hamiltonian and Lagrangian mechanics. Once we endorse categorical equivalence, we are forced (or perhaps, allowed) to recognize that there are actually a number of different theories that are standardly called "Hamiltonian mechanics." Hamiltonian mechanics on a cotangent bundle (as represented by the category $\mathbf{H a m}_{1}$ ), for example, is a different theory from Hamiltonian mechanics on a symplectic manifold (as represented by the category $\mathbf{H a m}_{2}$ ); the two theories are not categorically equivalent. ${ }^{21}$

We can only provide a definitive answer to the question "Are $T_{1}$ and $T_{2}$ equivalent?" once we have made the question clear and precise. And as the case of classical mechanics illustrates, there may be a number of different reasonable ways to go about making the question precise. It depends on what we mean by "equivalent" and on how we are understanding the theories in question. This immediately leads us to our first philosophical payoff. While perhaps unsurprising, it is nonetheless important to keep in mind when we consider

actually finding it. See Meehan (2017) for a careful discussion of this idea.

${ }^{21}$ Following the proof of Proposition 2, it is easy to verify that these theories are inequivalent. The intuition behind this verdict is something like the following. Hamiltonian mechanics on a symplectic manifold has "more models" than Hamiltonian mechanics on a cotangent bundle. The former theory can be used to describe to a wider range of physical systems than the latter theory. 
questions of equivalence.

Payoff 1. Whether one considers two theories to be equivalent depends both on the standard of equivalence that one employs, and on the precise way in which one understands the two theories in question.

In slogan form, this payoff can be summarized as follows: In order to say whether Hamiltonian and Lagrangian mechanics are equivalent, one must first say what one means by "Hamiltonian mechanics," "Lagrangian mechanics," and "equivalent." The same conclusion will obviously apply to any two theories, not just to these formulations of classical mechanics.

Payoff 1 does not imply that all standards of equivalence are on equal footing. As we have seen, many criteria make undesirable verdicts. We have good reason to avoid employing these criteria, and so it is important to be clear about exactly which standard of equivalence one is employing when answering a particular question of equivalence. Certain standards - like the model isomorphism criterion - simply do not provide us with as much interesting information about the relationship between the theories in question as other standards do. Payoff 1 is a plea for this kind of clarity in our discussions of equivalence.

There are two other general philosophical payoffs that I would like to conclude with. Philosophers of science have long been concerned with the following question:

How should we interpret what a physical theory says about the world? What is the content of a physical theory?

This question has come to be regarded as one of the most central questions in philosophy of science; van Fraassen (1986, p. 309), for example, calls it the "foundational question par excellence." I would like to suggest that this question is closely related to the question of when we should consider two theories to be equivalent. The final two payoffs are concerned with this relationship.

One particular answer to van Fraassen's question par excellence has recently become popular among philosophers of physics and metaphysicians. This answer proposes that we should interpret a physical theory by looking to the mathematical structure of the models of a physical theory and taking it "at face value." 22 This method of face value interpretation is most clearly described by North (2009, p. 78):

Take the mathematical formulation of a given theory. Figure out what structure is required by that formulation. [...] Infer that this is the fundamental structure of the theory. Go on to infer that this is the fundamental structure of the world, according to the theory.

This method of interpretation is motivated by a particularly strong form of "realism" about our physical theories. If one thinks — as realists are inclined to - that a physical theory provides us with a description of the world, then

\footnotetext{
${ }^{22}$ This turn of phrase is borrowed from Maudlin (2013), who makes a similar point to the one here, but without explicitly mentioning theoretical equivalence.
} 
it is natural to look to the mathematical structures employed by the models of the theory for this description. This thought is precisely what leads one to face value interpretation.

Face value interpretation has in recent years become the "received view" in philosophy of physics for how to best unravel the content of our physical theories. A particularly prominent example comes up in discussions of wavefunction realism in quantum mechanics. ${ }^{23}$ According to this view, quantum mechanics requires us to "take the space on which [wavefunctions] are defined, a configuration space of extraordinarily high dimension [...], as the arena in which events take place" (Myrvold, 2015, p. 3248). Our familiar three-dimensional physical space becomes secondary to configuration space. Indeed, some have claimed that "whatever impression we have [...] of living in a three-dimensional space [...] is somehow flatly illusory" (Albert, 1996, p. 277). One is led to wavefunction realism through a straightforward application of the face value method of interpreting physical theories. Since in the standard formulation of quantum mechanics one represents the state of a system with a wavefunction defined on a high-dimensional configuration space, one takes this mathematical structure at face value and infers that the physical space we live in is this high-dimensional configuration space (Albert, 1996, p. 277).

It is not my aim in this paper to offer a sustained argument against wavefunction realism. Rather, I would like to raise a concern about face value interpretation, the method of interpreting physical theories that led us to wavefunction realism in the first place. If we accept face value interpretation as our preferred method of interpreting our physical theories, then we are immediately forced to conclude - following the same route as North - that Hamiltonian and Lagrangian mechanics are inequivalent theories. Since the statespaces of these two theories come equipped with different structures, face value interpretation precludes us from recognizing an equivalence between them. Their models employ non-isomorphic mathematical structures, and they therefore must be describing the world in different ways. Lagrangian mechanics ascribes to the world the structure of a tangent bundle, while Hamiltonian mechanics ascribes to the world the structure of a cotangent bundle.

Face value interpretation forces us towards a particularly strict standard of equivalence between theories. Consider the theories from examples 1, 2, and 3 from the perspective of face value interpretation.

Example 1: (1,3) general relativity and (3,1) general relativity. If one takes the mathematical structures of these two theories at face value, then one is forced to recognize a difference between them. The former affirms that at every point of spacetime there are three linearly independent tangent vectors with negative length, while the latter denies this. These two theories therefore "say different things about the world," and a face value interpreter concludes that they are inequivalent.

\footnotetext{
${ }^{23}$ This position is endorsed by Albert (1996, 2013), Loewer (1996), Ney (2012, 2013a,b), and North (2013), among others. See the collected papers in Ney and Albert (2013) and the references therein.
} 
Example 2: Euclidean geometry with points and Euclidean geometry with lines. If we take the mathematical apparatus of these two theories at face value - and we think of them as describing the world - then the former theory says that the world is made up of points, while the latter says that the world is made up of lines. So we are forced to consider these two theories inequivalent too.

Example 3: General relativity and the theory of Einstein algebras. This case is perhaps the most dramatic of the three. A face value interpreter of these two theories will claim that they offer radically different descriptions of the world - according to the theory of Einstein algebras, the world has algebraic structure, while according to the standard formulation of general relativity, the world has the structure of a manifold with metric - and therefore consider them inequivalent.

As these examples illustrate, face value interpretation forces us to consider very few pairs of theories equivalent. In general, if we decide to interpret our physical theories by taking their mathematical structures at face value, then we are committing ourselves to a form of the model isomorphism criterion. Face value interpretation maintains that there is a strong sense in which the mathematical structure of a theory's models directly "mirrors" the structure of the world. (Recall, for example, the way in which wavefunction realists argue that the physical space we live in is configuration space.) If there is such a close relationship between the mathematical structure of a theory's models and the structure of the world, then theories whose models employ non-isomorphic structures must be saying different things about the world. This is precisely the content of the model isomorphism criterion. Insofar as we reject the model isomorphism criterion as our standard for equivalence between theories, therefore, we should also reject face value interpretation as an answer to van Fraassen's question par excellence. It forces us to make untenable verdicts in many simple cases of equivalence.

We can use exactly the kind of reasoning employed in this argument against face value interpretation as a new tool for deciding how best to interpret our physical theories. This idea is captured by the following payoff.

Payoff 2. When we commit to a particular method of interpreting our physical theories - that is, a method of reading off the content of a physical theory we are also committing ourselves to a standard of equivalence between theories.

If we have a method of interpretation in hand, we will naturally consider two theories to be equivalent when they have the same content according to this method. We can therefore evaluate proposed answers to van Fraassen's question par excellence by instead evaluating the standard for equivalence that they induce.

If we use Payoff 2 to reject face value interpretation, then one might worry that we are forced to adopt a radical form of "anti-realism." Fortunately, this is not the case. In fact, radically anti-realist methods of interpreting our physical 
theories commit us to standards of equivalence that are just as unsatisfactory as the model isomorphism criterion. We can therefore use Payoff 2 to reject them too. For example, Putnam (1977) famously suggested that we interpret our theories in such a way that any consistent theory could be true of the world. But this method of interpretation naturally leads us to judge any pair of consistent theories to be equivalent. Since any two consistent theories can be understood as true of the world, they must "say the same thing about the world." This standard for equivalence is clearly far too liberal; there are many pairs of consistent theories that we have good reason to consider inequivalent.

We should therefore look for a method of interpreting our physical theories that lies somewhere between face value interpretation and Putnam's radically anti-realist approach to interpretation. Our discussion here suggests a way to find such a method. ${ }^{24}$ I conjecture that the relationship between equivalence and interpretation is in fact even more robust than Payoff 2 suggests.

Payoff 3. When we commit to a standard for equivalence between physical theories, we are also committing ourselves to a method of interpreting - or unraveling the content of - our physical theories.

A standard of equivalence between physical theories goes hand-in-hand with a method of interpreting our physical theories. When we commit to a particular standard of equivalence between theories, we are also saying which features of our theories are significant or "contentful." I submit that the contentful features of a theory are precisely those that are invariant under the standard for equivalence that we adopt. Payoffs 2 and 3 together suggest that questions of equivalence, like the ones that we have been concerned with throughout this dissertation, are intimately related to van Fraassen's question par excellence. The moral of these two payoffs can be summarized as follows: We should look to cases of equivalent theories as a guide for how best to interpret our physical theories. We go a long way towards pinning down what a theory "says about the world" by pinning down which other theories "say the same thing."

\section{References}

Abraham, R. and J. E. Marsden (1978). Foundations of Mechanics. AddisonWesley.

Albert, D. Z. (1996). Elementary quantum metaphysics. In J. T. Cushing, A. Fine, and S. Goldstein (Eds.), Bohmian Mechanics and Quantum Mechanics: An Appraisal. Dordrecht: Kluwer.

Albert, D. Z. (2013). Wave function realism. In A. Ney and D. Z. Albert (Eds.), The Wave Function: Essays on the Metaphysics of Quantum Mechanics. Oxford University Press.

\footnotetext{
${ }^{24} \mathrm{~A}$ similar thought is expressed by Dewar (2016).
} 
Andréka, H., J. X. Madarász, and I. Németi (2005). Mutual definability does not imply definitional equivalence, a simple example. Mathematical Logic Quarterly 51(6), 591-597.

Bain, J. (2003). Einstein algebras and the hole argument. Philosophy of Science $70(5), 1073-1085$.

Barrett, T. W. (2015). On the structure of classical mechanics. The British Journal for the Philosophy of Science 66(4), 801-828.

Barrett, T. W. and H. Halvorson (2016a). Glymour and Quine on theoretical equivalence. Journal of Philosophical Logic 45(5), 467-483.

Barrett, T. W. and H. Halvorson (2016b). Morita equivalence. The Review of Symbolic Logic 9(3), 556-582.

Barrett, T. W. and H. Halvorson (2017a). From geometry to conceptual relativity. Forthcoming in Erkenntnis.

Barrett, T. W. and H. Halvorson (2017b). Quine's conjecture on many-sorted logic. Forthcoming in Synthese.

Borceux, F. (1994). Handbook of Categorical Algebra, Volume 1. Cambridge University Press.

Casado, C. M. M. (2008). A brief history of the mathematical equivalence between the two quantum mechanics. Latin-American Journal of Physics Education 2(2), 9.

Coffey, K. (2014). Theoretical equivalence as interpretative equivalence. The British Journal for the Philosophy of Science 65(4), 821-844.

Curiel, E. (2014). Classical mechanics is Lagrangian; it is not Hamiltonian. The British Journal for the Philosophy of Science 65(2), 269-321.

Dewar, N. (2016). What is it to interpret a theory? Unpublished manuscript.

Earman, J. (1977a). Leibnizian space-times and Leibnizian algebras. In R. E. Butts and J. Hintikka (Eds.), Historical and Philosophical Dimensions of Logic, Methodology and Philosophy of Science, pp. 93-112. Dordrecht: Reidel.

Earman, J. (1977b). Perceptions and relations in the monadology. Studia Leibnitiana 9(2), 212-230.

Earman, J. (1979). Was Leibniz a relationist? In P. French, T. Uehling, and H. Wettstein (Eds.), Midwest Studies in Philosophy, Volume 4, pp. 263-276. Minneapolis, MN: University of Minnesota Press.

Earman, J. (1986). Why space is not a substance (at least not to first degree). Pacific Philosophical Quarterly 67(4), 225-244. 
Earman, J. (1989a). Leibniz and the absolute vs. relational dispute. In N. Rescher (Ed.), Leibnizian Inquiries. A Group of Essays, pp. 9-22. Lanham, MD: University Press of America.

Earman, J. (1989b). World Enough and Space-Time. Boston: The MIT Press.

Eilenberg, S. and S. Mac Lane (1942). Group extensions and homology. Annals of Mathematics.

Eilenberg, S. and S. Mac Lane (1945). General theory of natural equivalences. Transactions of the American Mathematical Society.

Feynman, R. (1966). The development of the space-time view of quantum electrodynamics. Science 153(3737), 699-708.

Geroch, R. (1972). Einstein algebras. Comm. Math. Phys..

Glymour, C. (1971). Theoretical realism and theoretical equivalence. In PSA 1970, pp. 275-288. Springer.

Glymour, C. (1977). The epistemology of geometry. Noûs 11, 227-251.

Glymour, C. (1980). Theory and Evidence. Princeton University Press.

Glymour, C. (2013). Theoretical equivalence and the semantic view of theories. Philosophy of Science 80(2), 286-297.

Halvorson, H. (2011). Natural structures on state space. Manuscript.

Halvorson, H. (2012). What scientific theories could not be. Philosophy of Science 79(2), 183-206.

Halvorson, H. (2013). The semantic view, if plausible, is syntactic. Philosophy of Science 80(3), 475-478.

Halvorson, H. (2016). Scientific theories. In P. Humphreys (Ed.), The Oxford Handbook of Philosophy of Science, pp. 585-608. Oxford University Press.

Heisenberg, W. (1979). Letter of 8 June 1926. In K. v. M. A. Hermann and V. Weiskopf (Eds.), Wolfgang Pauli: Wissentschaftlicher Briefwechsel mit Bohr, Einstein, Heisenberg u.a., Volume 1 (1919-1929), pp. 328-329. Springer.

Hudetz, L. (2015). Linear structures, causal sets and topology. Studies in History and Philosophy of Modern Physics, 294-308.

Hudetz, L. (2016). Definable categorical equivalence. Manuscript.

Knox, E. (2011). Newton-Cartan theory and teleparallel gravity: The force of a formulation. Studies in History and Philosophy of Science Part B: Studies in History and Philosophy of Modern Physics 42(4), 264-275. 
Knox, E. (2014). Newtonian spacetime structure in light of the equivalence principle. The British Journal for the Philosophy of Science 65(4), 863-880.

Loewer, B. (1996). Humean supervenience. Philosophical Topics 24(101-127).

Mac Lane, S. (1968). Geometrical mechanics. Unpublished lecture notes.

Mac Lane, S. (1971). Categories for the working mathematician. Springer.

Maudlin, T. (2013). The nature of the quantum state. In A. Ney and D. Z. Albert (Eds.), The Wave Function: Essays on the Metaphysics of Quantum Mechanics. Oxford University Press.

Meehan, A. (2017). Physics and the art of reformulation. Manuscript.

Myrvold, W. C. (2015). What is a wavefunction? Synthese 192, 3247-3274.

Ney, A. (2012). The status of our ordinary three dimensions in a quantum universe. Nous 46, 525-560.

Ney, A. (2013a). Introduction. In A. Ney and D. Z. Albert (Eds.), The Wave Function: Essays on the Metaphysics of Quantum Mechanics. Oxford University Press.

Ney, A. (2013b). Ontological reduction and the wave function ontology. In A. Ney and D. Z. Albert (Eds.), The Wave Function: Essays on the Metaphysics of Quantum Mechanics. Oxford University Press.

Ney, A. and D. Z. Albert (Eds.) (2013). The Wave Function: Essays on the Metaphysics of Quantum Mechanics. Oxford University Press.

North, J. (2009). The 'structure' of physics: A case study. The Journal of Philosophy 106, 57-88.

North, J. (2013). The structure of a quantum world. In A. Ney and D. Z. Albert (Eds.), The Wave Function: Essays on the Metaphysics of Quantum Mechanics. Oxford University Press.

Putnam, H. (1977). Realism and reason. Proceedings and Addresses of the American Philosophical Association 50(6).

Quine, W. V. O. (1975). On empirically equivalent systems of the world. Erkenntnis 9(3), 313-328.

Rosenstock, S., T. W. Barrett, and J. O. Weatherall (2015). On Einstein algebras and relativistic spacetimes. Studies in History and Philosophy of Science Part B: Studies in History and Philosophy of Modern Physics 52, 309-316.

Rosenstock, S. and J. O. Weatherall (2016). A categorical equivalence between generalized holonomy maps on a connected manifold and principal connections on bundles over that manifold. Journal of Mathematical Physics 57(10). arXiv:1504.02401 [math-ph]. 
Rynasiewicz, R. (1992). Rings, holes and substantivalism: On the program of Leibniz algebras. Philosophy of Science 59(4), 572-589.

Schrödinger, E. (1926). On the relation between the quantum mechanics of Heisenberg, Born, and Jordan, and that of Schrödinger. Annalen der Physik 79, 734-756.

Sklar, L. (1982). Saving the noumena. Philosophical Topics, 89-110.

Swanson, N. and H. Halvorson (2012). On North's 'The structure of physics'. Manuscript.

Teh, N. and D. Tsementzis (2017). Theoretical equivalence in classical mechanics and its relationship to duality. Forthcoming in Studies in History and Philosophy of Modern Physics.

Tsementzis, D. (2015). A syntactic characterization of Morita equivalence. Manuscript.

van Fraassen, B. C. (1986). Aim and structure of scientific theories. Studies in Logic and the Foundations of Mathematics 114, 307-318.

Van Fraassen, B. C. (2014). One or two gentle remarks about Hans Halvorson's critique of the semantic view. Philosophy of Science 81(2), 276-283.

von Neumann, J. (1932). Mathematical Foundations of Quantum Mechanics. Princeton University Press.

Wald, R. M. (1984). General Relativity. Chicago.

Weatherall, J. O. (2016a). Are Newtonian gravitation and geometrized Newtonian gravitation theoretically equivalent? Erkenntnis 81(5), 1073-1091.

Weatherall, J. O. (2016b). Understanding gauge. Philosophy of Science 83(5), 1039-1049.

Weatherall, J. O. (2017). Category theory and the foundations of classical field theories. In E. Landry (Ed.), Forthcoming in Categories for the Working Philosopher. Oxford University Press.

\section{Appendix}

The purpose of this appendix is to prove Propositions 1, 2, and 3. In order to prove Proposition 1, we need the following lemma.

Lemma 1. F: $\mathbf{L a g}_{1} \rightarrow \mathbf{H a m}_{1}$ and $G: \mathbf{H a m}_{1} \rightarrow \mathbf{L a g}_{1}$ are functors. 
Proof. We show that $F$ is a functor. The argument for $G$ is perfectly analogous. It is trivial that $F$ maps objects of $\mathbf{L a g}_{1}$ to objects of $\mathbf{H a m}_{1}$. Let $T_{*} f:\left(T_{*} M_{1}, L_{1}\right) \rightarrow\left(T_{*} M_{2}, L_{2}\right)$ be an arrow in $\mathbf{L a g}_{1}$. We show that $F\left(T_{*} f\right)=$ $T^{*}\left(f^{-1}\right)$ is an arrow $\left(T^{*} M_{1}, E_{L_{1}} \circ \mathcal{L}_{L_{1}}^{-1}\right) \rightarrow\left(T^{*} M_{2}, E_{L_{2}} \circ \mathcal{L}_{L_{2}}^{-1}\right)$ in $\mathbf{H a m}_{1}$. One can easily compute both of the following

$$
\mathcal{L}_{L_{2}} \circ T_{*} f=T^{*}\left(f^{-1}\right) \circ \mathcal{L}_{L_{1}} \quad E_{L_{2}} \circ T_{*} f=E_{L_{1}}
$$

using the fact that $L_{2} \circ T_{*} f=L_{1}$. Since $\mathcal{L}_{L_{1}}$ and $\mathcal{L}_{L_{2}}$ are diffeomorphisms, these two equations together imply that $E_{L_{2}} \circ \mathcal{L}_{L_{2}}^{-1} \circ T^{*}\left(f^{-1}\right)=E_{L_{1}} \circ \mathcal{L}_{L_{1}}^{-1}$. The point*-transformation $T^{*}\left(f^{-1}\right)$ therefore preserves the Hamiltonians and is an arrow in $\mathbf{H a m}_{1}$. It follows immediately from the definition of $F$ that it respects composition and preserves identities.

Proposition 1. F: $\mathbf{L a g}_{1} \rightarrow \boldsymbol{H a m}_{1}$ and $G: \mathbf{H a m}_{1} \rightarrow \mathbf{L a g}_{1}$ are equivalences.

Proof. Theorem 1 implies that the functors $F$ and $G$ are "inverses" on the objects of $\mathbf{H a m}_{1}$ and $\mathbf{L a g}_{1} . F$ and $G$ are also "inverses" on the arrows of $\mathbf{H a m}_{1}$ and $\mathbf{L a g}_{1}$. This immediately implies that $F$ and $G$ are equivalences.

We now turn to Proposition 2. Our argument begins with a lemma that is perfectly analogous to Lemma 1.

Lemma 2. $F: \mathbf{L a g}_{1} \rightarrow \mathbf{H a m}_{2}$ is a functor.

Proof. One argues precisely as in Lemma 1 (using, in addition, the fact that point*-transformations preserve the canonical symplectic form).

Proposition 2. Neither $F: \mathbf{L a g}_{1} \rightarrow \mathbf{H a m}_{2}$ nor $G: \mathbf{H a m}_{2} \rightarrow \mathbf{L a g}_{1}$ is an equivalence.

Proof. We have already shown that $G$ is not a functor from $\mathbf{H a m}_{2}$ to $\mathbf{L a g}_{1}$. We now show that the functor $F: \mathbf{L a g}_{1} \rightarrow \mathbf{H a m}_{2}$ is neither essentially surjective nor full. Since there are symplectic manifolds that are not diffeomorphic to a cotangent bundle, one immediately sees that $F$ is not essentially surjective. To see that $F$ is not full, consider the cotangent bundle $T^{*} \mathbb{R} \cong \mathbb{R}^{2}$ with the symplectic form and Hamiltonian defined by

$$
\Omega_{a b}=\frac{1}{2}\left(\left(d_{a} x_{1}\right)\left(d_{b} x_{2}\right)-\left(d_{a} x_{2}\right)\left(d_{b} x_{1}\right)\right) \quad H\left(x_{1}, x_{2}\right)=x_{1}^{2}+x_{2}^{2}
$$

where $x_{1}$ and $x_{2}$ are the standard coordinate functions on $\mathbb{R}^{2}$. One can verify that $\Omega_{a b}$ is the canonical symplectic form on $T^{*} \mathbb{R}$ and that $H$ is a hyperregular Hamiltonian $H: T^{*} \mathbb{R} \rightarrow \mathbb{R}$. Theorem 1 implies that there is a hyperregular model of Lagrangian mechanics $\left(T_{*} \mathbb{R}, L\right)$ such that $F\left(T_{*} \mathbb{R}, L\right)=\left(T^{*} \mathbb{R}, \Omega_{a b}, H\right)$. We define the diffeomorphism $f: \mathbb{R}^{2} \rightarrow \mathbb{R}^{2}$ by $f:\left(x_{1}, x_{2}\right) \mapsto\left(-x_{2}, x_{1}\right)$. This map satisfies $f^{*}\left(\Omega_{a b}\right)=\Omega_{a b}$ and $H \circ f=H$, so $f:\left(T^{*} \mathbb{R}, \Omega_{a b}, H\right) \rightarrow$ $\left(T^{*} \mathbb{R}, \Omega_{a b}, H\right)$ is an arrow in $\mathbf{H a m}_{2}$. But since $f$ is not a point*-transformation, $f \neq F g$ for any arrow $g:\left(T_{*} \mathbb{R}, L\right) \rightarrow\left(T_{*} \mathbb{R}, L\right)$, the functor $F$ is not full. 
We conclude with a proof of Proposition 3. In the now-familiar manner, we begin with the following lemma.

Lemma 3. $G: \mathbf{H a m}_{3} \rightarrow \mathbf{L a g}_{2}$ is a functor.

Proof. It is trivial that $G$ maps objects of $\mathbf{H a m}_{3}$ to objects of $\mathbf{L a g}_{2}$. Let $T^{*} f:\left(T^{*} M_{1}, H_{1}\right) \rightarrow\left(T^{*} M_{2}, H_{2}\right)$ be an arrow in $\mathbf{H a m}_{3}$. This means that $\left(T^{*} f\right)_{*}\left(X_{H_{1}}\right)^{a}=\left(X_{H_{2}}\right)^{a}$. We need to show that show that $G\left(T^{*} f\right)=T_{*}\left(f^{-1}\right)$ is an arrow in $\mathbf{L a g}_{2}$ between $G\left(T^{*} M_{1}, H_{1}\right) \rightarrow G\left(T^{*} M_{2}, H_{2}\right)$. For convenience we will call $G\left(T^{*} M_{1}, H_{1}\right)=\left(T_{*} M_{1}, L_{1}\right)$ and $G\left(T^{*} M_{2}, H_{2}\right)=\left(T_{*} M_{2}, L_{2}\right)$.

We begin by showing that $H_{2} \circ T^{*} f=H_{1}+c$, where $c \in \mathbb{R}$ is some constant. Let $\Omega_{a b}$ be the canonical symplectic form on $T^{*} M_{1}$ and $\widehat{\Omega}_{a b}$ the canonical symplectic form on $T^{*} M_{2}$. We compute the following:

$$
\begin{aligned}
\Omega_{a b}\left(X_{H_{2} \circ T^{*} f}\right)^{a} & =d_{b}\left(H_{2} \circ T^{*} f\right) \\
& =\left(T^{*} f\right)^{*}\left(d_{b} H_{2}\right) \\
& =\left(T^{*} f\right)^{*}\left(\widehat{\Omega}_{a b}\left(X_{H_{2}}\right)^{a}\right) \\
& =\Omega_{a b}\left(T^{*} f\right)^{*}\left(X_{H_{2}}\right)^{a}=\Omega_{a b}\left(X_{H_{1}}\right)^{a}
\end{aligned}
$$

The first equality holds by the definition of $\left(X_{H_{2} \circ T^{*} f}\right)^{a}$, the second since pullbacks and exterior derivatives commute, the third by the definition of $\left(X_{H_{2}}\right)^{a}$, the fourth since $\left(T^{*} f\right)^{*}\left(\widehat{\Omega}_{a b}\right)=\Omega_{a b}$, and the fifth since $T^{*} f$ is an arrow in $\mathbf{H a m}_{3}$. Since $\Omega_{a b}$ is non-degenerate, these equalities imply that $\left(X_{H_{2} \circ T^{*} f}\right)^{a}=$ $\left(X_{H_{1}}\right)^{a}$. It immediately follows from this that $H_{2} \circ T^{*} f=H_{1}+c$ for some constant $c \in \mathbb{R}$. One uses this to show that

$$
\mathcal{H}_{H_{2}} \circ T^{*} f=T_{*}\left(f^{-1}\right) \circ \mathcal{H}_{H_{1}}
$$

The argument for (1) is a simple computation.

We conclude by computing the following, which implies that $T_{*}\left(f^{-1}\right)$ is an arrow in $\mathbf{L a g}_{2}$ between $\left(T_{*} M_{1}, L_{1}\right) \rightarrow\left(T_{*} M_{2}, L_{2}\right)$.

$$
\begin{aligned}
\left(T_{*}\left(f^{-1}\right)\right)_{*}\left(X_{L_{1}}\right)^{a} & =\left(\mathcal{H}_{H_{2}} \circ T^{*} f \circ \mathcal{H}_{H_{1}}^{-1}\right)_{*}\left(X_{L_{1}}\right)^{a} \\
& =\left(\mathcal{H}_{H_{2}}\right)_{*} \circ\left(T^{*} f\right)_{*} \circ\left(\mathcal{H}_{H_{1}}^{-1}\right)_{*}\left(X_{L_{1}}\right)^{a} \\
& =\left(\mathcal{H}_{H_{2}}\right)_{*} \circ\left(T^{*} f\right)_{*}\left(X_{H_{1}}\right)^{a} \\
& =\left(\mathcal{H}_{H_{2}}\right)_{*}\left(X_{H_{2}}\right)^{a} \\
& =\left(X_{L_{2}}\right)^{a}
\end{aligned}
$$

The first equality holds by (1), the second by simple properties of the pushforward, the third and fifth since $\mathcal{H}_{H_{1} *}\left(X_{H_{1}}\right)^{a}=\left(X_{L_{1}}\right)^{a}$ and $\mathcal{H}_{H_{2} *}\left(X_{H_{2}}\right)^{a}=$ $\left(X_{L_{2}}\right)^{a}$ (Abraham and Marsden, 1978, Theorem 3.6.2), and the fourth since $T^{*} f$ is an arrow in $\mathbf{H a m}_{3}$. This implies that $T_{*}\left(f^{-1}\right)$ is an arrow in $\mathbf{L a g}_{2}$. As in Lemma 1 one verifies that $G$ respects composition and preserves identities.

Proposition 3. Neither $F: \mathbf{L a g}_{2} \rightarrow \mathbf{H a m}_{3}$ nor $G: \mathbf{H a m}_{3} \rightarrow \mathbf{L a g}_{2}$ is an equivalence. 
Proof. We show that $G: \mathbf{H a m}_{3} \rightarrow \mathbf{L a g}_{2}$ is not full and that $F: \mathbf{L a g}_{2} \rightarrow \mathbf{H a m}_{3}$ is not a functor. Let $M=\mathbb{R}$ so that $T_{*} M \cong \mathbb{R}^{2}$. Consider the two Lagrangians $L_{1}: T_{*} M \rightarrow \mathbb{R}$ and $L_{2}: T_{*} M \rightarrow \mathbb{R}$ defined by

$$
L_{1}\left(x_{1}, x_{2}\right)=x_{2}^{2} \quad L_{2}\left(x_{1}, x_{2}\right)=2 x_{2}^{2}
$$

Both of these Lagrangians are hyperregular, so $\left(T_{*} M, L_{1}\right)$ and $\left(T_{*} M, L_{2}\right)$ are objects in $\mathbf{L a g}_{2}$. Theorem 1 implies that there are objects $\left(T^{*} M, H_{1}\right)$ and $\left(T^{*} M, H_{2}\right)$ in $\mathbf{H a m}_{3}$ such that $G\left(T^{*} M, H_{1}\right)=\left(T_{*} M, L_{1}\right)$ and $G\left(T^{*} M, H_{2}\right)=$ $\left(T_{*} M, L_{2}\right)$.

Now one can easily compute that $E_{L_{1}} \neq E_{L_{2}}+c$ for any constant $c \in \mathbb{R}$; indeed, $E_{L_{1}}=L_{1}$ and $E_{L_{2}}=L_{2}$. But one can also compute that $\left(X_{L_{1}}\right)^{a}=$ $\left(X_{L_{2}}\right)^{a}$. This second equality implies that the identity point ${ }_{*}$-transformation $T_{*}\left(1_{M}\right)$ is an arrow $\left(T_{*} M, L_{1}\right) \rightarrow\left(T_{*} M, L_{2}\right)$ in $\mathbf{L a g}_{2}$. On the other hand, $E_{L_{1}} \neq E_{L_{2}}+c$ implies that $H_{1} \neq H_{2}+c$ for any constant $c \in \mathbb{R}$, and so $\left(X_{H_{1}}\right)^{a} \neq\left(X_{H_{2}}\right)^{a}$. This means that $T^{*}\left(1_{M}\right)$ is not an arrow $\left(T^{*} M, H_{1}\right) \rightarrow$ $\left(T^{*} M, H_{2}\right)$ in $\mathbf{H a m}_{3}$, and $G$ is therefore not full. This argument immediately implies that $F$ is not a functor from $\mathbf{L a g}_{2}$ to $\mathbf{H a m}_{3} . F$ does not map the arrow $T_{*}\left(1_{M}\right):\left(T_{*} M, L_{1}\right) \rightarrow\left(T_{*} M, L_{2}\right)$ in $\mathbf{L a g}_{2}$ to an arrow in $\mathbf{H a m}_{3}$. 